\title{
Weed detection in 3D images
}

\author{
A. Piron · F. van der Heijden • M. F. Destain
}

Published online: 11 November 2010

(C) The Author(s) 2010. This article is published with open access at Springerlink.com

\begin{abstract}
Machine vision has been successfully used for mechanical destruction of weeds between rows of crops. Knowledge of the position of the rows where crops should be growing and the assumption that plants growing outside such positions are weeds may be used in such systems. However for many horticultural crops, the automatic removal of weeds from inside a row or bands of crops in which the weeds are mixed with plants in a random manner is not solved. The aim of this study was to verify that plant height is a discriminating parameter between crop and weed at early growth stages, as weeds and crops grow at different speeds. Plant height was determined by using an active stereoscopy technique, based on a time multiplexing coded structured light developed to take into account the specificities of the small scale scene, namely occlusion and thin objects, internal reflections and high dynamic range. The study was conducted on two carrot varieties sown at commercial density. Different weed species were present at the time of data acquisition. To accurately represent plant height taking into account the ground irregularities, a new parameter called 'corrected plant height' was computed. This parameter was the distance between plant pixels and the actual ground level under them obtained by fitting a surface and seen from a reconstructed point of view corresponding to a camera's optical axis perpendicular to the ridge plane. The overall classification accuracy without correction was $66 \%$ whereas it reached $83 \%$ by using the corrected plant height.
\end{abstract}

Keywords Weed detection · Plant height - Stereoscopy $\cdot$ Coded structured light

\footnotetext{
A. Piron $(\bowtie) \cdot$ M. F. Destain

Environmental Science and Technology Department, Gembloux Agricultural University,

Gembloux, Belgium

e-mail: piron.a@fsagx.ac.be

F. van der Heijden

Signals and Systems Group, Twente University, Enschede, The Netherlands
} 


\section{Introduction}

Autonomous robotic weed control systems may provide a means of reducing agriculture's current dependency on herbicides, improving its sustainability and reducing its environmental impact. In the design of a robotic system, detection and identification of weeds under the highly variable conditions usually found in agricultural fields remains the greatest challenge (Slaughter et al. 2008). Two problems have to be considered. The first one is the detection of weeds from soil with the goal of eliminating weeds between the rows or between widely spaced individual crop plants. The second problem aims to recognize weeds mixed with plants in random positions. This is a complex task as the scenes are unstructured, the distribution of the crop in the rows is irregular and there is no a priori knowledge concerning the weeds present.

Several attributes, such as topological properties (area, invariant moments and curvature features), are found useful in weed identification. To detect weeds from corn plants, Tellaeche et al. (2008) extracted cells from RGB images, each cell being described by two area-based measuring relationships. Søgaard (2005) developed a method for machine classification of weed species based on active shape models. Berge et al. (2008) used shape parameters to detect broad-leaved patches in cereal crop. Success rate was between 84 and $90 \%$. These shape-based methods generally achieve high recognition rates under ideal conditions, i.e. when the shape of the entire leaf is well displayed (no occlusion) (Slaughter et al. 2008). A large number of studies have investigated the use of colour or spectral reflectance techniques for species identification. Feyaerts and van Gool (2001) developed a spectrograph with a low spectral resolution $(35 \mathrm{~nm})$ and used it to discriminate beets from five weed species. Classification accuracy was good (up to $86 \%$ ), however, six narrow spectral bands were necessary which is impractical for in-field work. Vrindts et al. (2002) used a hyperspectral machine vision system with very narrow spectral bands to classify sugar beet and weed plants in the field with similar limitations. Nieuwenhuizen et al. (2007) used color information to detect volunteer potatoes in sugar beet fields. The results were very variable with classification rates ranging from 49 to $97 \%$ in different fields. Piron et al. (2008) used a combination of three wide-band interference filters to detect weeds located within carrot rows and found a classification accuracy of only $72 \%$. To obtain robust and more accurate classification, knowledge about leaf orientation and their relative heights is necessary, to get more complete visual information available to the recognition process (Lee and Slaughter 2004). 3D information about the leaves is useful to separate overlapping objects and to analyze spectral anomalies in relation to their location in the canopies (Lee et al. 1999). Sanchez and Marchant (2000) described the possibility of detecting weeds by a fixed threshold on plant height on stereoscopic images of plants in laboratory conditions. Nielsen et al. (2004) studied the detection of weeds among tomato plants by analyzing stereoscopic images acquired in the field by a trinocular camera. The distinction between crop and weeds was based on three different methods: simple per-pixel threshold on distance from camera to plant pixels, analysis of connected blobs' height histograms and analysis of those same blobs after watershed segmentation. The Authors acknowledged the negative effect of ground irregularity on classification. Other plant properties have also been studied with stereoscopic images. He et al. (2003) used stereoscopic images acquired by a binocular camera to evaluate average plant height and leaf area (other parameters were evaluated too but are not relevant to this review), on potted transplants. Andersen et al. (2005) studied the possibility of computing plant geometric properties such as plant height and leaf area on stereoscopic images acquired with a binocular camera, on isolated potted plants. They showed that those characteristics can be 
determined using stereovision but specify that the acquisition method needs to be validated for in-field conditions and for more complex plant structures. Plant height could thus possibly be used for weed detection among young carrots but this hypothesis must be tested for in-field conditions.

The most well-known method to acquire 3D information is passive stereovision; it was for example used in the studies reported above. This method is based on viewing the scene from two or more points of view and then finding correspondences between the different images in order to triangulate the $3 \mathrm{D}$ position. When the scene does not contain singular points such as corners, or when it presents discontinuities or thin structures (which is the case for in-field weed detection), correspondences are difficult to find. With regards to the difficulty of acquiring good quality stereoscopic data of plants by stereovision, Nielsen et al. (2007) developed a framework to compare and tune stereoscopic algorithms on virtual images of various plants.

To acquire 3D information, other imaging methods are available such as active stereoscopic methods based on structured light projection (Pagès et al. 2005). In this case, a camera is used to image the projection of a given pattern (for example a laser plane) on the scene. 3D information manifests itself in the deformations of the imaged pattern compared to the projected one (Salvi et al. 2004). Coded structured light can be considered as an evolution of structured light techniques and is based on the projection of bi-dimensional patterns by using light projectors, the patterns containing a form of encoding of spatial information. Those methods improve the reliability and quality of the $3 \mathrm{D}$ information compared to passive stereovision techniques. For this study, coded structured light was chosen because it is better suited to scenes with holes, occlusions and quick depth changes compared to non-coded structured light.

The objective of this paper was to verify that plant height is a discriminating parameter between young carrot crop and weeds, as weeds and crops grow at different speeds. To achieve this goal, a stereoscopic acquisition method based on coded structured light was developed taking into account scene specificities. A "corrected plant height" parameter was computed from the acquired stereoscopic images and used for classification of weed and crop.

The structure of the paper is as follows: first, the acquisition, coding and decoding strategies are given and justified with regard to the scene and acquisition device specificities. Second, actual implementation and measurements description are outlined. Finally, plant classification based on the "corrected plant height" parameter is described.

\section{Materials and methods}

Acquisition, coding and decoding strategies

For coded structured light, which uses light projectors such as in this case a video projector, several patterns have been proposed to realize the correspondence between the image plane and the projected image with accuracy. Pattern projection techniques are classified according to their coding strategies: direct codification, neighborhood codification and time-multiplexing (Salvi et al. 2004). In direct codification, where the gray or color value of each pixel contains all the information necessary to retrieve its spatial position in the pattern, the sensitivity to noise is very high and it is necessary to perceive and identify the whole spectrum of projected colors. Furthermore, those techniques are not particularly well suited for non-uniformly colored objects. In spatial neighborhood 
techniques, the codeword that labels a certain point of the pattern is obtained from a neighborhood of the points around it. In our scene, numerous occlusions and discontinuities are present which prevent the identification of neighborhood information. A timemultiplexing technique was thus chosen. It consists in projecting successively a set of patterns onto the scene. The codeword for a given pixel is thus formed by the sequence of illumination values for that pixel across the projected patterns.

The stereoscopic imaging system was to be combined with a color acquisition device using artificial lighting designed to select optimal spectral bands for weed detection (Piron et al. 2008). This system was composed of a black and white camera and a filter wheel that dictated the use of monochromatic patterns. A binary code was chosen, which meant that only two illumination levels were used, which were coded as 0 (black intensity) and 1 (full illumination). This small codeword basis allowed more robust decoding (Salvi et al. 2004).

Overview of scene and acquisition material-related problems and existing solutions

The small-scale agronomic scenes presented several characteristics that influenced the techniques used. However, for each characteristic no single solution existed, and reciprocally, each solution did not apply to a single problem. This is summarized in Table 1.

The scene presented a high dynamic range, i.e. it had regions high in reflectance and others low in reflectance (Robertson et al. 1999): the soil, particularly when it was wet, had a considerably lower reflectance than the plants (Fig. 1). In this case, when projecting patterns at low illumination intensities, the signal-to-noise ratio of the system in the soil areas decreased and therefore depth from low reflective regions could not be obtained. On the other hand, when projecting high illumination intensity patterns, depth from regions with high reflectance could not be recovered, due to pixel saturation (Salvi et al. 2004). Furthermore, internal reflections were present, i.e. a certain, high reflectance part of the

Table 1 Presentation of scene specificities relevant to coded structured light and associated solutions

\begin{tabular}{|c|c|c|c|c|c|c|}
\hline & & & \multicolumn{4}{|c|}{ Solutions } \\
\hline & & & \multicolumn{2}{|l|}{ Codes } & \multirow{2}{*}{$\begin{array}{l}\text { Patterns } \\
\text { Pseudo- } \\
\text { random } \\
\text { patterns }\end{array}$} & \multirow{2}{*}{\begin{tabular}{|l} 
Acquisition \\
$\begin{array}{c}\text { Exposure } \\
\text { fusion }\end{array}$
\end{tabular}} \\
\hline & & & $\begin{array}{c}\text { Long binary codes, } \\
\text { Lowly correlated, } \\
\text { decoded by } \\
\text { correlation }\end{array}$ & $\begin{array}{l}\text { Per-pixel } \\
\text { decoding }\end{array}$ & & \\
\hline \multirow{5}{*}{ 䓪 } & Material & $\begin{array}{c}\text { Limited } \\
\text { projector depth } \\
\text { of field } \\
\end{array}$ & & & & \\
\hline & \multirow{4}{*}{ Scene } & $\begin{array}{l}\text { High dynamic } \\
\text { range scene }\end{array}$ & & & & \\
\hline & & $\begin{array}{l}\text { Internal } \\
\text { reflections }\end{array}$ & & & & \\
\hline & & Thin objects & & & & \\
\hline & & Occlusions & & & & \\
\hline
\end{tabular}


Fig. 1 Detail of a scene with a projected pattern

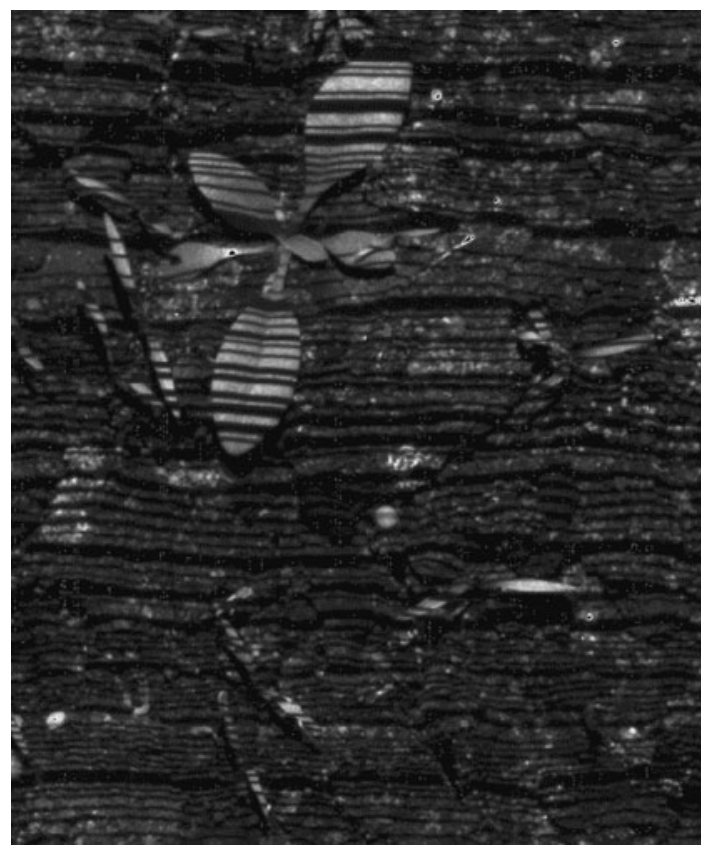

scene illuminated another, low reflectance one, being a possible source of noise in the signals. This phenomenon was worsened by the high dynamic range of the scene.

To solve the variation of reflectance problem, in scenes with no internal reflections, existing solutions consist in varying the intensity distribution of the projection patterns. Lu and Cho (2005) proposed a pattern intensity control based on acquiring a fully illuminated image of the scene to adjust automatically to surface reflectance. Wu et al. (2006) used reflectance ratios from two patterns and high dynamic range imaging.

In the presence of high dynamic range scenes with internal reflections, Scharstein and Szeliski (2003) found that a reliable way of thresholding pixels was to project both the code pattern and its inverse. Each pixel can be labeled according to whether the pattern or its inverse appears brighter. This technique has to be completed by selecting the exposure setting that yields the largest absolute difference between two illuminations.

The second problematic peculiarity of the canopy concerned the numerous depth discontinuities within plant layers and between plants and ground, leading to numerous occlusions (part of the scene either invisible to the camera or not illuminated by the projector). To solve occlusion problems in binary stripe patterns, Scharstein and Szeliski (2003) used per pixel segmentation as described above in the previous section.

The third problem related to the scene concerned the very thin structures exhibited by the plants. In some cases, the leaves are barely larger than either the camera or video projector pixel size. Gorpas et al. (2007) noted the lack of works in the 3D optical measurement field dealing with small objects $(<1 \mathrm{~cm}$ diameter $)$.

Besides these problems related to the scene, peculiarities of the equipment could influence the coding and decoding strategy. In our application, the depth of field of the projector was inferior to the measurement volume $(120 \mathrm{~mm})$. This problem is not specifically addressed in the literature but, as the blurry pattern could be interpreted as noise in the signal, it could be argued that coding schemes aimed at improving signal robustness 
were relevant. According to Salvi et al. (2004), solutions comprised error code inclusion in the patterns, redundant codes or spatio-temporal analysis.

Implemented solutions

Coding: long binary codes, lowly correlated, decoded by correlation

The nature of the code was chosen to give robust results. Weakly correlated codes with a minimum Hamming distance ${ }^{1}$ of 8 (empirically determined) between any pair of codes were used. The codes were decoded by correlating the signal received by a single camera pixel over time with all possible signals. As correlation also gave a measure of the reliability of the decoding, it could be used to remove spurious measurements by applying a threshold.

The length of the code had to satisfy three conditions: to be adapted to the minimum Hamming distance requirement, to allow the encoding of full projector resolution (768 lines) and to give good decoding results. The third criterion was decisive. The length of the code used was 22 bits. Hamming code error correction was not used. The small codeword basis (binary) also allowed more robust decoding as stated earlier. Those characteristics are meant to deal with the limited projector depth of field and internal reflections since both problems could be considered as noise in the signals perceived by the camera.

\section{Coding: per-pixel decoding}

The signals received at each pixel were decoded without taking into account neighboring pixels, since there were a large number of discontinuities. This also allowed obtaining depth information for fine plant leaves and bracts structures that could be of a size similar to camera or projector pixel size.

\section{Patterns: pseudo random patterns}

Usually, in time-multiplexing, binary code techniques, the projected images are comprised of black and white bands of large then finer width. The wider bands cause problems when the scene is prone to internal reflections because the large areas illuminated by a white band of the pattern reflect on areas illuminated by a dark area of the pattern.

To avoid this problem, the patterns used were pseudo-random (e.g. without apparent structure). Such patterns are usually associated in structured light techniques that use spatial neighborhood coding techniques to spatially encode information (e.g. Spoelder et al. 2000). In this case, however, the more homogeneous illumination given by a pseudorandom pattern is used to minimize the effects of internal reflections. Figure 2 presents the link from the codes to the pattern.

\section{Acquisition: exposure fusion}

On the basis of work of Scharstein and Szeliski (2003), several exposures were used to acquire a single image of each pattern. However, the implementation of the high dynamic range imaging was different and based on exposure fusion (Robertson et al. 1999). Four exposures of each pattern were taken at different exposure times and "blended" using the following process.

\footnotetext{
1 The Hamming distance between two codes is the number of corresponding bits that are different.
} 


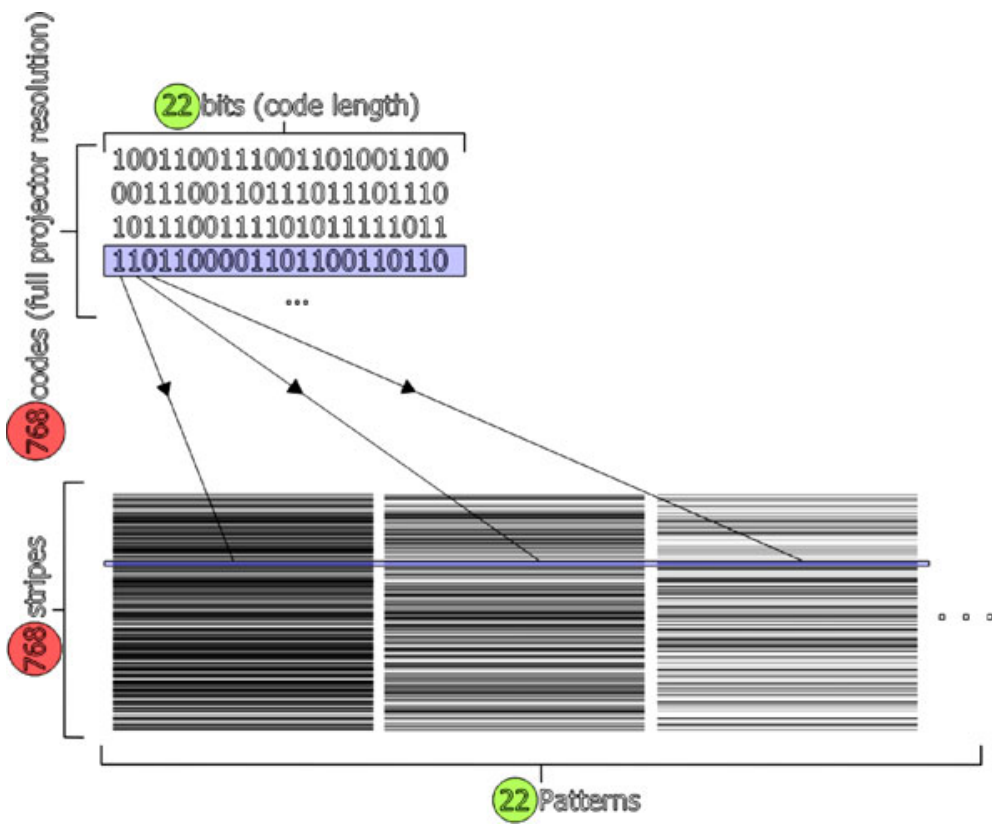

Fig. 2 Principle of the transposition of coded structured light codes to patterns

On each image, it was determined which pixel was neither under- or overexposed (two thresholds). The first image in descending exposure time order was used as the base image onto which information from all the other images were added, in descending exposure time order. After this step, the information from the next ones were added to the first image, one by one, for corresponding pixels that satisfied the exposure condition, by scaling it for the second image exposure time. Pixels that did not appear correctly exposed in at least two images were not taken into account. This last step allowed the detection of occlusions and specular reflections. The high dynamic range acquisition also allowed to have a strong signal to noise ratio for all pixels of the image.

The exposure times and the total number of exposures were determined empirically on potted plants. It was found by trial and error that four exposure times $(0.6,0.3,0.07$ and $0.01 \mathrm{~s})$ were necessary because of the large variations of soil reflectance and of certain plant species.

Stereoscopic acquisition implementation

\section{Image acquisition}

The stereoscopic device was combined with an existing camera used in a previous study to allow measurement of depth over multi-spectral images. The multi-spectral acquisition was based on a black and white camera (Vector International C-cam BCI 51.3 megapixels, Leuven, Belgium) coupled with a filter wheel (Figs. 3, 4). The spectral bands of interest for weed detection were centered at 450, 550 and $700 \mathrm{~nm}$. For plant-ground segmentation, only the first and last ones were used. The coded structured light patterns were acquired without a filter in front of the camera. 


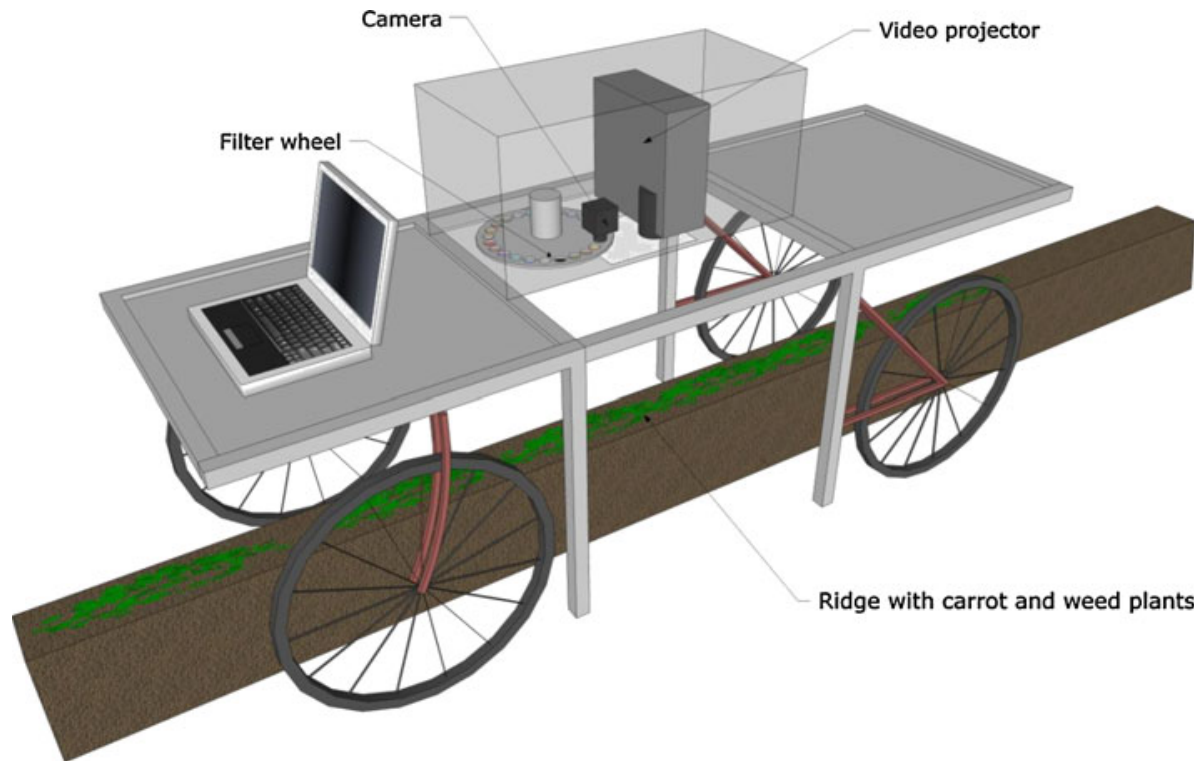

Fig. 3 Schematic view of the acquisition device (natural light shielding and artificial lighting parts not represented)

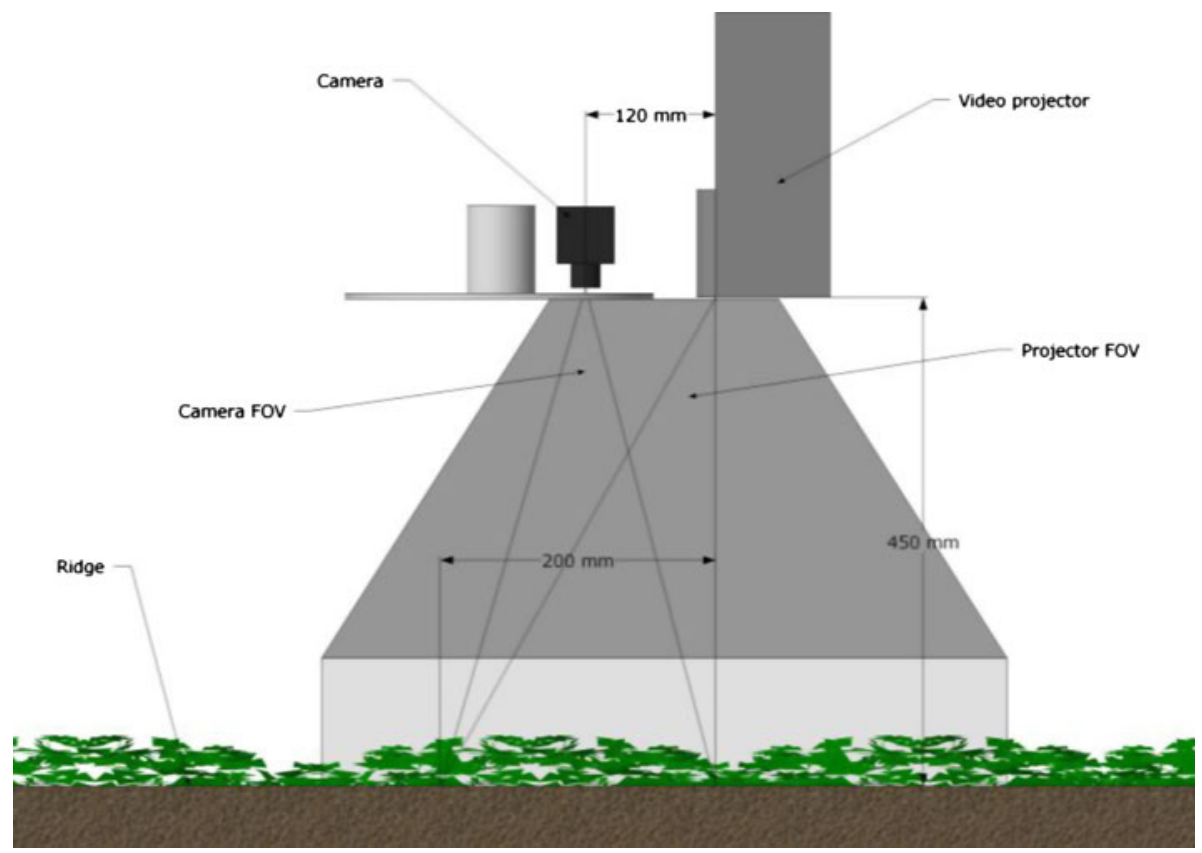

Fig. 4 Lateral schematic view of the projector/camera system showing the respective fields of view (FOV) and approximate relative positions of the elements. The trapezoidal element under the projector/camera system corresponds to the natural light shielding and artificial lighting parts that are omitted for clarity's sake in Fig. 3 
Table 2 Summary of acquired data

\begin{tabular}{llll}
\hline $\begin{array}{l}\text { Date of data } \\
\text { acquisition }\end{array}$ & $\begin{array}{l}\text { Days after } \\
\text { sowing }\end{array}$ & $\begin{array}{l}\text { Soil surface } \\
\text { state }\end{array}$ & $\begin{array}{l}\text { Acquired } \\
\text { stereoscopic images }\end{array}$ \\
\hline 22 May 2006 & 21 & Wet & 4 \\
29 May 2006 & 28 & Wet & 4 \\
31 May 2006 & 30 & Wet & 9 \\
07 June 2006 & 37 & Dry & 7 \\
09 June 2006 & 39 & Dry & 4 \\
\hline
\end{tabular}

The video projector (OPTOMA EP719, $1024 \times 768$ resolution) was chosen for its ability to be focused on very close objects.

A mobile support frame (Fig. 3) was designed to allow acquisition of top-down images of the field scene (approximately 200 by $250 \mathrm{~mm}$ ). The scenes were shielded from natural light. The study was conducted on two carrot varieties without distinction, Nerac F1 and Namur F1. Approximately 200 linear metres of rows were mechanically sown at a density of 10-15 seeds per 100 linear mm over a $50 \mathrm{~mm}$ width which is a common commercial planting density. Several species of weeds were naturally present in the field and others were manually introduced. The main species were the following at the time of data acquisition: Sonchus asper L., Chenopodium sp., Cirsium sp., Merurialis M. perennis, Brassica sp. and Matricaria maritima. Images of carrot-sown ridges were taken over a period of 19 days during which soil moisture varied from dry to wet according to the weather (see Table 2). This timeframe is typical for manual weeding for carrots. Indeed, early weed detection can increase yields and weed elimination becomes increasingly difficult with plant growth. A total of 51 multi-spectral images were acquired at random locations in the parcel. The number of images acquired per day varied according to meteorological conditions: strong winds made the acquisition of images difficult because of the movement of plants and/or camera.

\section{Projector and camera calibration}

The calibration of the camera-projector system was done using the Zhang technique from the Intel OpenCV library. This model is physics-based and uses several parameters to describe the camera-projector system: focal lengths, pixel sizes, lenses principal points and four distortion parameters per lens. As it is a widespread method, we will only describe the specific adaptations performed.

A chessboard pattern was used for calibration. The camera lens user-defined parameters (focal distance and diaphragm opening) were set for multi-spectral and coded structured light patterns acquisition. Those settings limited the depth of field of the camera for acquisition of calibration images to barely more than the measurement volume but this did not pose any apparent calibration problem. A first attempt to illuminate the chessboard pattern using the projector for camera calibration resulted in very short exposure times for the camera. Due to the nature of the projection technique (DLP), short exposure times gave images with varying intensity over time. The DLP projection technique is based on an array of movable micro mirrors, each corresponding to a pixel of the projected image that are used to reflect (or not) light through the projector's lens. The fast movement of those mirrors provides a gray scale image.

In the projector used, the color is produced by a color filter wheel placed between the lamp of the projector and the micro-mirror array. The wheel has three filters (red, green, 
blue). To create the illusion of a color image, three successive images corresponding to the three-color channels are projected successively. The relatively low speed of the wheel creates the intensity fluctuations in the images captured by the camera. This problem also appeared when calibrating the projector by projecting a chessboard pattern on a flat calibration surface. The solution used was to average a certain number of exposures until the grey levels stopped varying significantly. When calibrating the projector by projecting a chessboard pattern on a flat calibration surface, the limited depth of field of the projector did not interfere with the chessboard corner detection algorithm.

Since we use per-pixel decoding, there was no need for following the epi-polar constraint or rectifying the images prior to decoding.

Plant classification

\section{Computing of plant height parameter}

The distance of the plants relative to the measurement device is not a good indicator of their actual heights if the position from the device to the ground varies or if the ground presents high roughness, especially if the plants are young and therefore of small size (Nielsen et al. 2004). This is schematically illustrated in Fig. 5.

Accurate 3D information about the soil was obtained by coded structured light imaging.

A corrected plant height parameter expressed as the distance between plant pixels and the actual ground level was computed. It was obtained by fitting a surface through ground pixels in order to interpolate 3D information missing due to either camera, projector or scene occlusions, and computing a new point of view corresponding to the optical axis perpendicular to the ridge plane of the camera.

The process is described in Fig. 6. In the first stage, plant and ground pixels were segmented by performing quadratic discriminant analysis on two spectral bands $(1,2)$. In the second stage, two surfaces were fitted through the soil pixels. First, one plane (3) was adjusted using a RANSAC (RANdom SAmple Consensus) algorithm (Fischler and Bolles 1981). The distance threshold parameter to determine whether a data point was an inlier or not was manually adjusted from terrain observations. Second, a triangle-based cubic interpolated surface (4) was fitted, using the grid-data function of Matlab. Because this function produced a surface that passed through all specified points, it was very sensitive to spurious pixels resulting from the imperfect segmentation between plants and ground. Furthermore, those pixels were frequently present at the limit between plants and ground,

Fig. 5 Left: distance measured during 3D acquisition. Right: plant height required for plant classification
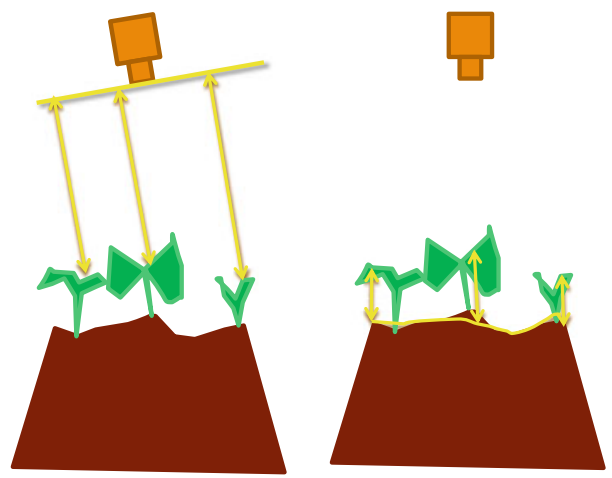


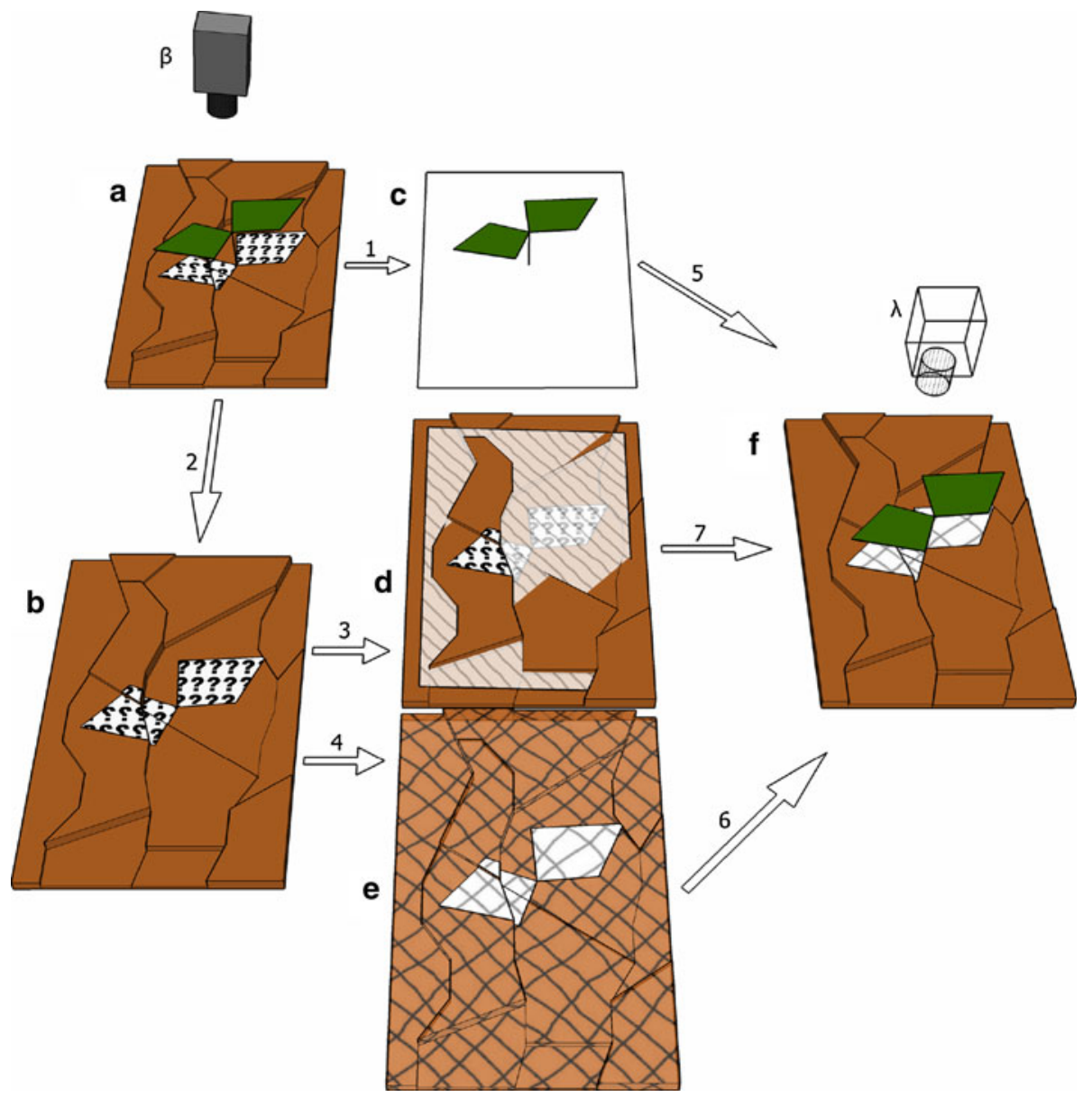

Fig. 6 Plant height parameter determination process. a Multispectral stereoscopic image, b soil image, c plant image, $\mathbf{d}$ plane fitted through soil, e surface fitted through soil, $\mathbf{f}$ final image for height determination with reconstructed soil and ridge and camera plane aligned, $\beta$ position of real camera relative to scene, $\lambda$ position of virtual camera corresponding to the viewpoint where ridge and camera plane are aligned

which was the border of the regions that were of interest for the plant height determination. To avoid this problem, the borders of those regions were eroded by a round structuring element of diameter 3 pixels. The plant and soil pixels (the latter with the interpolated pixels obtained in operation 4 since the ground under the plants was not visible from the camera, and not all points seen by the camera were illuminated by the projector) were then put back together $(5,6)$. Finally, the orientation of the fitted plane was used to rotate the data in space so as to align the plane normal with a virtual camera $(\lambda)$ optical axis. This is done to allow correction for measurement device placement.

\section{Classification}

The objectives of the classification were to classify weeds from crop and to analyze the value of introducing height information concerning the ground. Two parameters were used 
in the classification. The first one was for each plant pixel either the distance between the plant pixel and the reconstructed soil surface underneath (plant height parameter) or the distance from the plant pixel to the camera. The second was, in both cases, the number of days after sowing, which allowed to adapt the threshold height level to the rapid growth of the young plants over the data acquisition period.

Quadratic discriminant analysis (QDA) was used for data classification.

Manually segmented data (crop and weed classes) were used as a training set for the classifier. To ensure equal representation of both classes in the classification process, the number of points in the training set had to be the same for each class. Since there were less weed data points than plant points, we first applied a random sampling to the carrot data points to have the same amount of data in each class. Due to the large amount of measurement points, computations could be very long, so three subsequent random samplings were applied to both classes to extract $10 \%$ (for each sampling) of the pixels of the carrot leaves and the same amount of weeds, creating three new data subsets. Resubstitution validation was used to verify that the three data subsets gave the same $\mathrm{CA}$. Those random samplings were also done as a precaution: resubstitution validation can be prone to overfitting but it is rarely an issue in the case of a large amount of measurement points (Michie et al. 1994).

The criterion used to evaluate the efficiency of the feature combination was the classification accuracy. It is the percentage of observations correctly classified, whether for each class or for both classes together.

QDA and resubstitution validation might not be the optimal methods, but the goal was to compare feature sets and not to find the best classification method.

\section{Results and discussion}

Stereoscopic acquisition

The stereoscopic data acquisition gave highly detailed images with dense information and few decoding errors, as can be seen in Fig. 7 and 8. After thresholding on the minimum correlation value (visually determined), the amount of correctly decoded points over all images was $95 \%$, taking into account occlusions. The occlusions areas are visible as low correlation in Fig. 8: they correspond to the areas not illuminated by the projector because of shadows of other features. Decoding errors appeared to be randomly distributed over the images. For both soil and plants, the correlation for the signal decoding was very high (see Fig. 8), although better for plants than for soil.

The structures of plants with finely dissected leaves such as M. maritima (Fig. 7 top right) were clearly visible. The great variability in height of the ground could also be seen in those examples despite the high reflectance difference between plants and ground. Soil moisture didn't apparently influence decoding results.

Plant classification

The classification accuracy was compared when combining, on the one hand, non-corrected plant height and number of days after sowing and, on the other hand, corrected plant height parameter and number of days after sowing. It was found that the camera position and the ground irregularities greatly influenced the classification accuracies: using the corrected plant height parameter improved significantly the classification results (Table 3). 

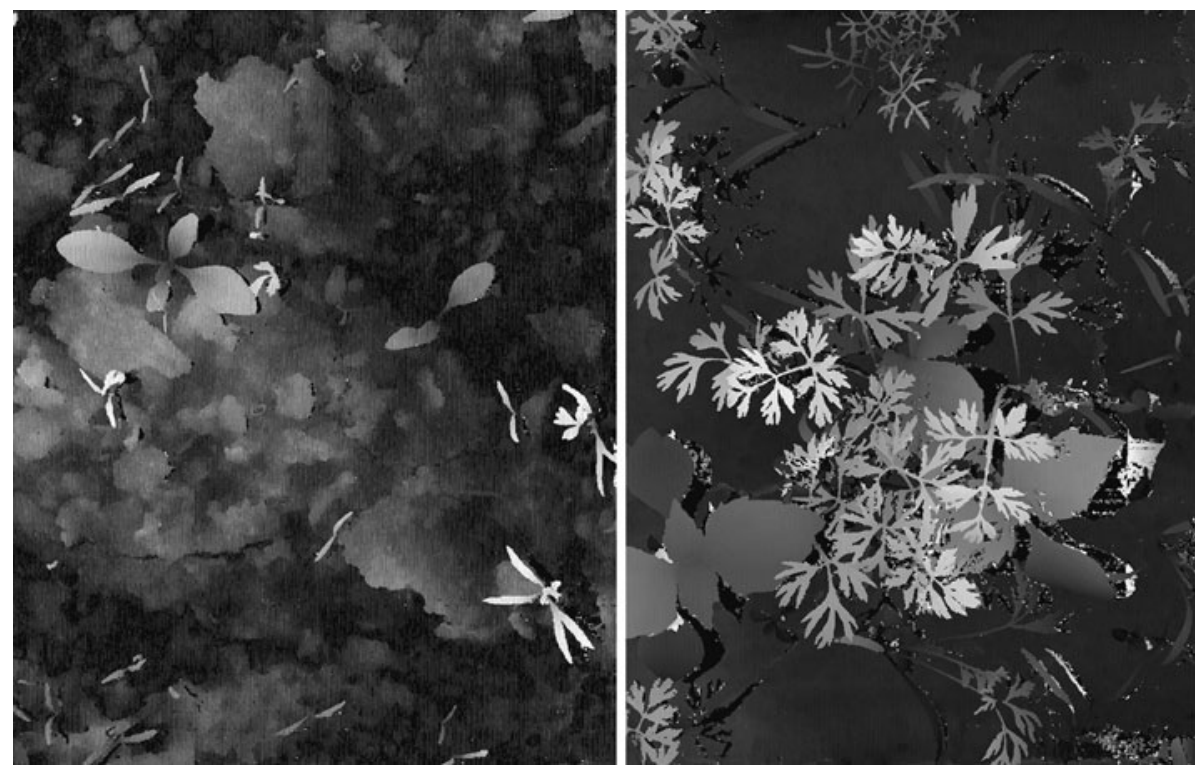

Fig. 7 Crops of stereoscopic depth images showing the high variability in ground flatness and multiple weed species. Lighter colors correspond to points closer to the camera

Fig. 8 Example crop of image of maximum correlation for each pixel

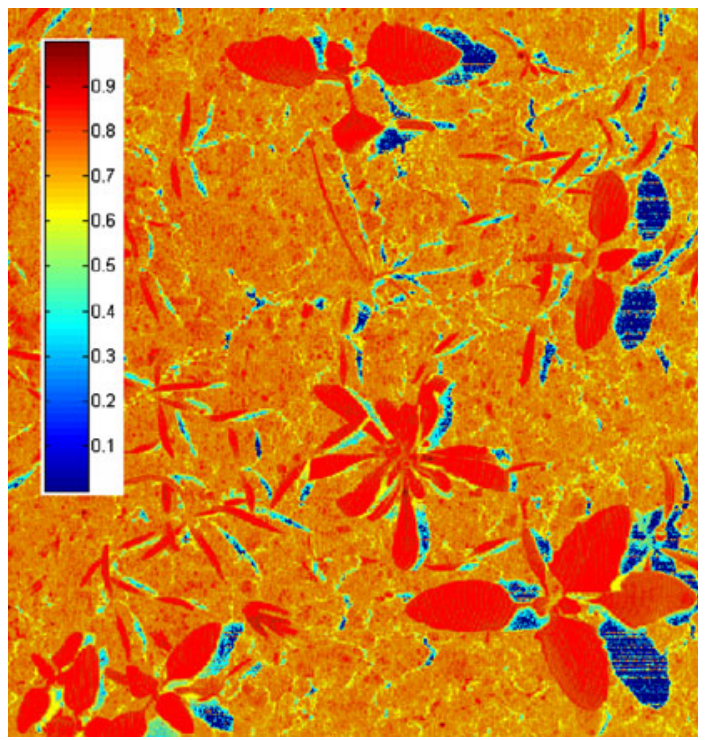

The overall classification accuracy without correction was $66 \%$. Taking into account the corrected plant height parameter, the overall classification accuracy reached $83 \%$. For the carrot class, the improvement when going from the non-corrected height parameter to the plant height parameter was smaller than for the weed class. This could be explained by the central position of the carrot plants on the ridge and the smoother surface state of the soil in that area, due to the sowing apparatus. Furthermore, since the camera was centered 
Table 3 Classification accuracy (\%) for each height parameter

\begin{tabular}{|c|c|c|c|}
\hline & \multirow{2}{*}{$\begin{array}{l}\text { Plant-camera } \\
\text { distance }\end{array}$} & \multicolumn{2}{|l|}{ Parameter } \\
\hline & & $\begin{array}{l}\text { Plant-camera distance } \\
\text { corrected for camera position }\end{array}$ & $\begin{array}{l}\text { Plant height } \\
\text { parameter }\end{array}$ \\
\hline Overall & 66 & 67 & 83 \\
\hline Carrots & 75 & 75 & 85 \\
\hline Weeds & 57 & 59 & 80 \\
\hline
\end{tabular}

on the carrot seed line, height variation of the camera relative to the ground due to inclination of the supporting frame was of less importance for that part of the image, at least laterally. The lower classification accuracy of weeds compared to carrots could be due to the greater variability of this class (several species) and their presence anywhere on the ridge.

As can be seen from Table 3, camera position correction only has a small positive effect on classification accuracy for weeds. The low improvement is likely due to the measurement conditions: the mobile frame was manually moved around the field and the field was not disturbed by tractor use. Bigger improvements are expected if the measurement equipment was mounted on a tractor and done in a field where the ground is more irregular due to tractor use.

\section{Conclusion}

A coded, structured-light method tailored for acquiring high quality stereoscopic images of small-scale field scenes was described. The method was compatible with an existing camera based on a filter wheel and comprised a DLP video projector. The coded structured-light method was based on a time-multiplexing approach with a binary codeword basis. The scenes studied had several specificities that had to be taken into account for the acquisition method: presence of numerous occlusions and thin objects, high dynamic range, internal reflections. In addition, the small scale of the scene meant that the depth of field of the projector was insufficient. To solve those problems, several approaches were used in the coding, patterns and acquisition methods: the codes were long binary codes, lowly correlated and decoded by correlation. The decoding was done on a per-pixel basis. Pseudo-random patterns were used and the acquisition used exposure fusion to deal with the high dynamic range and internal reflections of the scene. Acquisition results were qualitatively and quantitatively evaluated. It was found that the acquisition methods gave dense stereoscopic data of ground and plants even when the latter exhibited fine structures. This method is well suited for data acquisition for research purposes but is quite slow due to the large amount of images to acquire. For inline acquisition other, faster, techniques have to be considered.

The acquired stereoscopic data were classified to differentiate weeds from crop. This was done by using quadratic discriminant analysis with two combinations of two parameters. The first parameter was, for each combination, the number of days after sowing. The second parameter was, in one case, the distance from measurement device to plant pixels and, in the other case, a corrected plant height parameter that took into account camera placement and ground irregularities. The classification accuracy was only $66 \%$ in the first case while it reached $83 \%$ in the second case. This result showed the importance 
determining a parameter describing plant height by acquiring stereoscopic information about the ground as well as the plants in order to differentiate plants from weeds and not to only base the discrimination on measurement device-plant distance. For this to be efficient however there has to be a difference of height between crop and weed species.

Acknowledgment This project is funded by the "Ministère de la Région wallonne—DGTRE", project "RECADVEN" no EPH3310300R049F/41J81.

Open Access This article is distributed under the terms of the Creative Commons Attribution Noncommercial License which permits any noncommercial use, distribution, and reproduction in any medium, provided the original author(s) and source are credited.

\section{References}

Andersen, H. J., Reng, L., \& Kirk, K. (2005). Geometric plant properties by relaxed stereo vision using simulated annealing. Computers and Electronics in Agriculture, 49(2), 219-232.

Berge, T., Aastveit, A., \& Fykse, H. (2008). Evaluation of an algorithm for automatic detection of broadleaved weeds in spring cereals. Precision Agriculture, 9(6), 391-405.

Feyaerts, F., \& van Gool, L. (2001). Multi-spectral vision system for weed detection. Pattern Recognition Letters, 22, 667-674.

Fischler, M. A., \& Bolles, R. C. (1981). Random sample consensus: A paradigm for model fitting with applications to image analysis and automated cartography. Communications of the ACM, 24(6), 381-395.

Gorpas, D., Politopoulos, K., \& Yova, D. (2007). A binocular machine vision system for three-dimensional surface measurement of small objects. Computerized Medical Imaging and Graphics, 31, 625-637.

He, D. X., Matsuura, Y., Kozai, T., \& Ting, K. C. (2003). A binocular stereovision system for transplant growth variable analysis. Applied Engineering in Agriculture, 19(5), 611-617.

Lee, W. S., \& Slaughter, D. C. (2004). Recognition of partially occluded plant leaves using a modified watershed algorithm. Transactions of the ASA, 47(4), 1269-1280.

Lee, W. S., Slaughter, D. C., \& Giles, D. K. (1999). Robotic weed control system for tomatoes. Precision Agriculture, 1(1), 95-113.

Lu, C., \& Cho, G. (2005). Projection pattern intensity control technique for 3-D optical measurement. Optics Express, 13, 106-114.

Michie, D., Spiegelhalter, D. J., \& Taylor, C. C. (Eds.). (1994). Machine learning, neural and statistical classification. Chichester, UK: Ellis Horwood.

Nielsen, M., Andersen, H. J., Slaughter, D. C., \& Giles, D. K. (2004). Detecting leaf features for automatic weed control using trinocular stereo vision. In D. Mulla (Ed.), Proceedings of the 7th International Conference on Precision Agriculture, University of Minnesota, MN, USA. CD-ROM.

Nielsen, M., Andersen, H. J., Slaughter, D. C., \& Granum, E. (2007). Ground truth evaluation of computer vision based 3D reconstruction of synthesized and real plant images. Precision Agriculture, 8(1-2), $49-62$.

Nieuwenhuizen, A. T., Tang, L., Hofstee, J. W., Müller, J., \& van Henten, E. J. (2007). Colour based detection of volunteer potatoes as weeds in sugar beet fields using machine vision. Precision Agriculture, 8(6), 267-278.

Pagès, J., Salvi, J., Collewet, C., \& Forest, J. (2005). Optimized De Bruijn patterns for one-shot shape acquisition. Image and Vision Computing, 23(8), 707-720.

Piron, A., Leemans, V., Kleynen, O., Lebeau, F., \& Destain, M.-F. (2008). Selection of the most efficient wavelength bands for discriminating weeds from crop. Computers and Electronics in Agriculture, 62, $141-148$.

Robertson, M. A., Borman, S., \& Stevenson, R. L. (1999). Dynamic range improvement through multiple exposures. In Proceedings of the International Conference on Image Processing 99, Kobe, Japan, 24-28 October (vol. 3, pp. 159-163). IEEE Computer Society.

Salvi, J., Pages, J., \& Battle, J. (2004). Pattern codification strategies in structured light systems. Pattern Recognition, 37(4), 827-849.

Sanchez, A. J., \& Marchant, J. A. (2000). Fusing 3D information for crop/weeds classification. In A. Sanfeliu, J. J. Villanueva, M. Vanrell, R. Alquezar, T. Huang, \& J. Serra (Eds.), Proceedings of the 
15th International Conference on Pattern Recognition (ICPR'00) (Vol. 4, p. 4295). Los Alamitos, CA: IEEE Computer Society Press.

Scharstein, D., \& Szeliski, R. (2003). High-accuracy stereo depth maps using structured light. In C. Dyer \& P. Perona (Eds.), Proceedings of IEEE Computer Society Conference on Computer Vision and Pattern Recognition (CVPR 2003), Madison, WI, Vol. 1, pp. 195-202.

Slaughter, D. C., Giles, D. K., \& Downey, D. (2008). Autonomous robotic weed control systems: A review. Computers and Electronics in Agriculture, 61, 63-78.

Søgaard, H. T. (2005). Weed classification by active shape models. Biosystems Engineering, 93(3), 271-281.

Spoelder, H. J. W., Vos, F. M., Petriu, E. M., \& Groen, F. C. A. (2000). Some aspects of pseudo random binary array-based surface characterization. IEEE Transactions on Instrumentation and Measurement, 49(6), 1331-1336.

Tellaeche, A., Burgos-Artizzu, X. P., Pajares, G., \& Ribeiro, A. (2008). A vision-based method for weeds identification through the Bayesian decision theory. Pattern Recognition, 41(2), 521-530.

Vrindts, E., De Baerdemaeker, J., \& Ramon, H. (2002). Weed detection using canopy reflection. Precision Agriculture, 3(1), 63-80.

Wu, A. C., Madan, S., \& Dana, K. J. (2006). Projector-camera structured light using photometric ratios. In C. Jaynes \& G. Welch (Eds.), Proceedings of the Procams International Workshop on ProjectorCamera Systems workshop of the 2006 IEEE Conference on Computer Vision and Pattern Recognition, New York (pp. 15-16). 